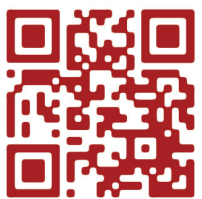

\title{
Will this trial change my practice? ACCOAST - early loading with a novel P2Y12 inhibitor in patients with an acute coronary syndrome
}

\author{
Rodney De Palma ${ }^{1}$, MD; Stefan James², MD; Peter Jüni ${ }^{3}$, MD; Thomas Cuisset ${ }^{4 *}$, MD \\ 1. The Heart Hospital, Cardiology Department, London, United Kingdom; 2. Department of Medical Sciences, Cardiology, and \\ Uppsala Clinical Research Center, Uppsala University, Uppsala, Sweden; 3. Division of Clinical Epidemiology and Biostatistics, \\ Institute of Social and Preventive Medicine, University of Bern, Berne, Switzerland; 4. Département de Cardiologie, CHU La \\ Timone, France
}

\begin{abstract}
Abbreviations
CABG coronary artery bypass grafting

CI confidence interval

DAPT dual antiplatelet therapy

ESC European Society of Cardiology

HR hazard ratio

Hs-TnT high-sensitivity troponin T

MI myocardial infarction

NNH number needed to harm

NSTE ACS non-ST elevation acute coronary syndromes

PCI percutaneous coronary intervention

RRR relative risk reduction

STEMI ST elevation myocardial infarction
\end{abstract}

The contemporary paradigm for managing high-risk NSTE ACS involves an invasive strategy with the use of antithrombotic therapy to reduce ischaemic complications and recurrent adverse cardiovascular events while simultaneously avoiding bleeding complications. Pre-treatment (before coronary angiography) with P2Y12 inhibitors and aspirin has been shown to be of no advantage in lowrisk patients; conversely, CLARITY-TIMI 28 and TRITON-TIMI
38 suggested a benefit for pre-treatment in very high-risk patients (acute STEMI) $^{1,2}$. Pre-loading in NSTE ACS with clopidogrel has been based on the positive results of the randomised CURE study ${ }^{3}$, which included medically managed patients who did not undergo systematic catheterisation and an observational subset (PCI-CURE) who did receive PCI ( $21 \%$ of the cohort at 10 days). This introduction along with the objectives of the session was enunciated by Thomas Cuisset and served as the context for the Comparison of Prasugrel at PCI or Time of Diagnosis of Non-ST Elevation Myocardial Infarction (ACCOAST) trial $^{4}$ and the subsequent discussion at EuroPCR.

The invited panellists ranged from those with active experience of clinical cardiology from around Europe (Andreas Baumbach [UK], Marco Valgimigli [The Netherlands], Rod Stables [UK], Franz-Josef Neumann [Germany], Stefan James [Sweden]) to a cardiovascular epidemiologist (Peter Jüni [Switzerland]) and the principal investigator of ACCOAST himself (Gilles Montalescot [France]).

Franz-Josef Neumann framed the discussion with a real-life case history from his hospital. Rod Stables delivered an evidence-based overview of antithrombotic therapy of ACS past and present. He

\footnotetext{
*Corresponding author: Département de Cardiologie, CHU La Timone, France

E-mail:Thomas.CUISSET@ap-hm.fr
} 
ended with four questions for the audience and practitioners to ponder. "(1) Do such studies reflect the totality of the patients? (2) Do such studies capture all adverse events? (3) Are there significant angio-PCI time delays in the studies? (4) Is there an evidence-based choice for ACS patients who do not receive PCI?"

Indeed, the chair questioned the relevance of ACCOAST to his general interventional practice, as he frequently received patients over the weekend or on call overnight, who could not undergo immediate or even urgent PCI for medical reasons or due to the logistics of the weekend.

Montalescot re-acquainted the audience with the data (Figure 1), presented in 2013, of this large $(n=4,038)$ international $(>50 \%$ recruited from Europe) multicentre, randomised study. In a NSTEMI population the investigators set out to evaluate the superiority of systematic upstream ("pre-loading") DAPT (aspirin and prasugrel) using a split loading dose of prasugrel $30 \mathrm{mg}$ followed by a further $30 \mathrm{mg}$ in the cathlab if PCI was to be performed versus upstream placebo followed by $60 \mathrm{mg}$ in the cathlab if PCI was performed. Invasive coronary angiography with or without ad hoc PCI was mandated between 2 and 48 hours post-randomisation. One panellist asked whether this was "really a study looking at prasugrel or early invasive therapy". The trialist admitted it was the latter also, but benefit of pre-treatment was assessed in the study as well. Peter Jüni posed a question on the efficacy of study allocation concealment which was not described in the original publication. Furthermore, he commented that "the trial had an $80 \%$ power to detect a $24 \%$ RRR with 400 primary endpoint events. The trial was "terminated prematurely but 398 events had occurred by that time making it still adequately powered". The primary composite endpoint of reduced cardiovascular death, myocardial infarction, stroke, urgent revascularisation, or glycoprotein IIb/IIIa inhibitor bail-out through seven days from randomisation was not met $(10 \%$ vs. $9.8 \%$ ) by prasugrel and, importantly, there were more major bleeds (access, non-access as well as non-CABG-related) than in the reference arm $(2.6 \%$ and $1.4 \%$, respectively, for TIMI major; HR 1.9, 95\% CI: $1.19-3.02, p=0.0006, \mathrm{NNH} 84$ ), causing the

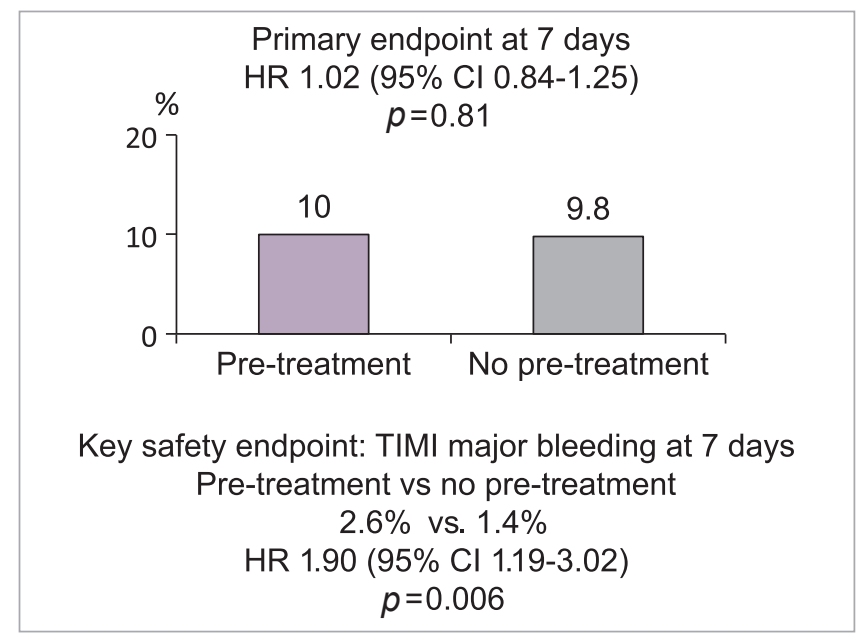

Figure 1. Summary slide taken from PCR trials book. monitoring board to terminate the study prematurely. In pre-specified higher risk subgroups, including patients treated with PCI, similar results were found.

Does this mean that all pre-loading with P2Y12, as supported by current ESC guidelines (1C recommendation for "as soon as possible"), is unnecessary and harmful? Peter Jüni praised the refreshing trial design "which was different to studies typically orientated to academic institutions" and felt that "the increasingly rapid access to acute cardiology services, pre-hospital assessment and initial treatment by paramedic staff, and the need for CABG in a proportion of patients make this a relevant question for practising cardiologists worldwide". This view was supported by delegates in the audience.

The cohort studied was representative of a broad NSTEMI population and included $25 \%$ at high risk as calculated by a GRACE score $>140$ points. All were troponin-positive and $54 \%$ had ischaemic ECG changes. The bleeding risk was moderate in the group as a whole (median CRUSADE score 33). The median time to invasive coronary angiogram was an impressive 4.3 hours. Although Gilles Montalescot countered the criticism from the audience that this did not reflect real-world practice by pointing out these transfer times were "similar to other contemporary NSTE ACS studies such as TIMACS and OASIS 7", Baumbach and Stables nevertheless insisted that "external validity was limited as for many units there are delays due to transfer logistics to a PCI-capable unit, apparent clinical stability and comorbidity". These delays without DAPT may risk ischaemic injury that has been seen previously with a "cooling off" strategy ${ }^{5}$. Stables wondered if the endpoint criteria had included troponin assessment. Montalescot confirmed that they had. The apparent "lack of difference most likely reflects early access to invasive assessment and treatment" said Baumbach. "The excess early bleeding hazard, however, underlined the importance of ischaemic and bleeding risk profiles". A member of the audience commented that systematic transfer of "troponin-positive" patients who are not true NSTEMI (type $2 \mathrm{MI}$ ) exposes them to pre-treatment bleeding risk of potent antithrombotic therapy without benefit and so may have contributed to the outcomes recorded. In the ACCOAST trial a medical strategy was pursued in $25.1 \%$ of the patients.

All panellists commented that the results could not be extrapolated to the other potent P2Y12, ticagrelor. Indeed, the main question was to discuss if ACCOAST showed no benefit of pre-treatment with strong P2Y12 blockers including ticagrelor ("strategy study") and no benefit of pre-treatment with prasugrel specifically ("drug study").

How can this study apply to daily practice? A poll of the audience indicated clearly that, while the ACCOAST was interesting as it challenged the guidelines, it would not change practice for the majority of their units owing to an average 48-hour or more delay from diagnosis to invasive therapy. However, in centres with short delay $(<48 \mathrm{hrs}$ ) to the cathlab for NSTEMI, panellists recognised the validity of the ACCOAST study. Bringing directly to the cathlab, however, as a NSTE ACS, then waiting to define the coronary anatomy before thienopyridine loading seemed more prudent and was supported by the study results with the caveat that we 
must wait for more data on ticagrelor pre-treatment. For those cases where the diagnosis is not clearly NSTE ACS then this study did support withholding additional antiplatelet therapy until the coronary anatomy is defined. Rod Stables still made the case for more flexibility in whether to pre-treat or not, as he would be "more comfortable pre-treating a 40-year-old man with a low risk of bleeding than an elderly woman with a small build". Stefan James and Marco Valgimigli, wrapping up the session, pointed out that "rather than dogmatically applying a policy of systematic pre-treatment or not, a more appropriate conclusion may be that we need to individualise antithrombotic therapy much more and focus on bringing appropriately triaged high-risk NSTE ACS to the cathlab sooner". As a final thought, the chairman summed up the feelings of many in the audience by declaring that there remained "confusion following the data from ACCOAST but at a higher level".

\section{Conflict of interest statement}

T. Cuisset has received consulting and lecture fees from Astra Zeneca and Eli Lilly. The other authors have no conflicts of interest to declare.

\section{References}

1. Sabatine MS, Cannon CP, Gibson CM, López-Sendón JL, Montalescot G, Theroux P, Claeys MJ, Cools F, Hill KA, Skene AM, McCabe $\mathrm{CH}$, Braunwald E; CLARITY-TIMI 28 Investigators. Addition of clopidogrel to aspirin and fibrinolytic therapy for myocardial infarction with ST-segment elevation. $N$ Engl J Med. 2005;352:1179-89.

2. Montalescot G, Wiviott SD, Braunwald E, Murphy SA, Gibson CM, McCabe CH, Antman EM; TRITON-TIMI 38 investigators. Prasugrel compared with clopidogrel in patients undergoing percutaneous coronary intervention for ST-elevation myocardial infarction (TRITON-TIMI 38): double-blind, randomised controlled trial. Lancet. 2009;373:723-31.

3. Yusuf S, Zhao F, Mehta SR, Chrolavicius S, Tognoni G, Fox KK; Clopidogrel in Unstable Angina to Prevent Recurrent Events Trial Investigators. Effects of clopidogrel in addition to aspirin in patients with acute coronary syndromes without ST-segment elevation. N Engl J Med. 2001;345:494-502.

4. Montalescot G, Bolognese L, Dudek D, Goldstein P, Hamm C, Tanguay JF, ten Berg JM, Miller DL, Costigan TM, Goedicke J, Silvain J, Angioli P, Legutko J, Niethammer M, Motovska Z, Jakubowski JA, Cayla G, Visconti LO, Vicaut E, Widimsky P; ACCOAST Investigators. Pretreatment with prasugrel in non-ST-segment elevation acute coronary syndromes. $N$ Engl J Med. 2013;369:999-1010.

5. Neumann FJ, Kastrati A, Pogatsa-Murray G, Mehilli J, Bollwein H, Bestehorn HP, Schmitt C, Seyfarth M, Dirschinger J, Schömig A. Evaluation of prolonged antithrombotic pretreatment ("cooling-off" strategy) before intervention in patients with unstable coronary syndromes: a randomized controlled trial. JAMA. 2003;290:1593-9. 